\title{
ESPÉCIES E PREVALÊNCIA DAS INFESTAÇÕES POR CARRAPATOS EM CÃES DE RUA DA CIDADE DE PORTO ALEGRE, RS, BRASIL
}

\author{
SPECIES AND PREVALENCE OF TICKS INFESTATIONS ON STRAY DOGS \\ IN PORTO ALEGRE CITY, RS, BRAZIL
}

\author{
Vera Lúcia Sardá Ribeiro ${ }^{1}$ Maria Angélica Weber ${ }^{2} \quad$ Liane Oliveira Fetzer $^{3}$ \\ Carla Rosane Bacêdo de Vargas ${ }^{4}$
}

RESUMO

O objetivo deste trabalho foi identificar as espécies de carrapatos que parasitam os cães da área urbana de Porto Alegre e conhecer suas prevalências nesta população. Para isso, foram examinados 450 cães de rua de Porto Alegre, colhidos os carrapatos nos animais infestados e estes identificados ao estereomicroscópio por espécie e por estágio. Dentre os animais examinados, 52,44\% albergavam carrapatos e destes, 93,22\% estavam parasitados por Rhipicephalus sanguineus, 2,97\% por Amblyomma aureolatum e $3,81 \%$ pelas duas espécies simultaneamente. Do total de carrapatos colhidos, a espécie mais abundante nos animais foi $\boldsymbol{R}$. sanguineus representando 99,43\% desse total; foram encontrados instares de todos os estágios parasitários de $\boldsymbol{R}$. sanguineus e só do estágio adulto de A. aureolatum.

Palavras-chave: espécies de carrapatos, prevalência, cães de rua, Porto Alegre, Rio Grande do Sul, Brasil.

SUMMARY

The purpose of this study was to identify and to know the prevalence of ticks recovered from dogs of urban area of Porto Alegre, RS. Four hundred and fifty stray dogs were examined

\begin{abstract}
and the ticks found were identified according to the species and parasitic stage. A percentage of $52.44 \%$ of those dogs were infested with ticks, among these, $93.22 \%$ were parasitized by Rhipicephalus sanguineus, $2.97 \%$ by Amblyomma aureolatum and $3.81 \%$ by both species. $R$. sanguineus was the more prevalent species among infested animals representing $99.43 \%$ of the total number of ticks sampled. All parasitic stages of $\boldsymbol{R}$. sanguineus were present on the stray dogs but only the adult stage of $\boldsymbol{A}$. aureolatum was found.
\end{abstract}

Key words: ticks species, prevalence, stray dogs, Porto Alegre, Rio Grande do Sul, Brazil.

\section{INTRODUÇÃO}

Nos últimos anos, em Porto Alegre, cada vez são mais freqüentes os casos de infestações dos cães por carrapatos. Estes, em virtude dos seus hábitos alimentares hematófagos, têm grande importância médica e veterinária, pois podem transmitir uma variedade de agentes patogênicos tanto para os animais como para o homem.

\footnotetext{
${ }^{1}$ Médico Veterinário, MsC., Professora Adjunta, Departamento de Patologia Clínica Veterinária, Faculdade de Veterinária, Universidade Federal do Rio Grande do Sul, Av. Bento Gonçalves, 9090. 91540-000, Porto Alegre, RS, Brasil. Fone: (051) 3166103. FAX (051) 3391513. (Autora para correspondência).

${ }^{2}$ Biólogo, MsC., Coordenadora do Centro de Controle de Zoonoses (CCZ), Centro de Vigilância em Saúde (CVS), Secretária Municipal de Saúde (SMS), Prefeitura Municipal de Porto Alegre (PMPA).

${ }^{3}$ Biólogo, CCZ, CVS, SMS, PMPA.

${ }^{4}$ Médico Veterinário, CCZ, CVS, SMS, PMPA.
} 
Os cães podem ser parasitados por várias espécies de carrapatos (FLECHTMANN, 1990) e dependendo da região, uma ou mais espécies podem ser mais comuns (DIPEOLU, 1975; RIVOSECCHI $\boldsymbol{e} \boldsymbol{t}$ al., 1980; MASSARD et al., 1981; ENCINAS GRANDES, 1986; HORAK et al., 1987; CORNACK \& O'ROURKE, 1991; MUMCUOGLU et al., 1993a/b).

No Rio Grande do Sul, Brasil em levantamento da fauna ixodológica, realizado por FREIRE (1972), foram identificados nos cães nove espécies de carrapatos: Amblyomma tigrinum, $\boldsymbol{A}$. ovale, $A$. aureolatum ( $=$ A. striatum $),$ A. cajennense, A. maculatum, A. humerale, A. incisum, Boophilus microplus e Rhipicephalus sanguineus.

MASSARD $\boldsymbol{e t}$ al. (1981) ao estudarem as parasitoses de cães, no período 1976-1980, no Rio Grande do Sul e em alguns outros estados brasileiros, como Rio de Janeiro, Espirito Santo e São Paulo, verificaram que os cães dessas regiões eram parasitados, quando criados em áreas rurais, pelos carrapatos do grupo "ovale" (A. ovale, A. aureolatum e A. tigrinum) e por A. cajennense (exceto no Rio Grande do Sul); nas áreas urbanas, o R. sanguineus foi a única espécie encontrada.

Quando a distribuição geográfica é considerada, e no que concerne à cidade de Porto Alegre, RS, são citadas as seguintes espécies parasitando os cães: R. sanguineus (ARAGÃO, 1936; CORRÊA, 1947; OLIVEIRA \& RIBEIRO, 1982/1983; RIBEIRO et al., 1994), A. ovale (CORRÊA, 1947; FREIRE, 1972) e A. aureolatum (FREIRE, 1972; OLIVEIRA \& RIBEIRO, 1982/1983; RIBEIRO et al., 1994). Apesar destas três espécies serem parasitos comuns entre os cães (FLECHTMANN, 1990), em Porto Alegre, até alguns anos atrás, as suas prevalências eram baixas. CORRÊA (1947) ao estudar as espécies e os índices entomológicos dos artrópodos que infestavam os cães de Porto Alegre, encontrou um único exemplar fêmea de $\boldsymbol{R}$. sanguineus em um dos cães $(1,29 \%)$ e dois exemplares machos em cada um dos dois cães $(2,60 \%)$ parasitados por A. ovale dentre os 77 cães examinados. OLIVEIRA \& RIBEIRO (1982/1983) ao examinarem 129 cães de rua em Porto Alegre, no período de maio/ 81 a abril/82, também encontraram um número pequeno de animais parasitados com $\boldsymbol{R}$. sanguineus e com $\boldsymbol{A}$. aureolatum; cada uma das espécies ocorreu somente em $1,55 \%$ dos animais examinados. No cão, o $\boldsymbol{R}$. sanguineus é o transmissor da Babesia canis (protozoário), do Hepatozoon canis (protozoário), do Eperythrozoon canis (riquétsia) e da Erlichia canis (riquétsia) (SWANGO et al., 1992).

GODDARD (1989) investigou a relação entre o número crescente de casos registrados de erliquiose humana devido à infecção por $\boldsymbol{E}$. canis e o aumento do parasitismo humano por $\boldsymbol{R}$. sanguineus levando ao aumento desta doença. Para o homem, ele pode transmitir ainda outras espécies de riquétsias, tal como a Rickettsia rickettsii e a $\boldsymbol{R}$. conorii, agentes causais respectivamente da Febre Maculosa das Montanhas Rochosas no México e na América do Sul e da Febre Botonosa na região mediterrânea do sul da Europa e da África do Norte (SWANGO et al., 1992). O cão também é susceptível à ação dessas riquétsias, apresentando na doença sinais clínicos similares aos que ocorrem no homem (GREENE \& PHILLIPS, 1984), no entanto, no cão elas se apresentam em geral subclinicamente (ACHA \& SZYFRES, 1986).

O cão tem um importante papel na epidemiologia dessas doenças que ocorrem no homem, pois é ele quem leva os carrapatos infectados para o ambiente humano (ACHA \& SZYFRES, 1986). Quando os cães coabitam com o homem, os carrapatos freqüentemente se desprendem e se espalham pela habitação, sendo encontrados em grande número nas paredes, debaixo dos móveis etc; esta situação favorece o contato do homem com o carrapato (KRINSKY, 1983).

Embora muitos autores, como MOREL \& VASSILIDES (1962), considerem que o $\boldsymbol{R}$. sanguineus parasita o homem só de maneira excepcional, o número crescente de casos documentados de parasitismo humano por esta espécie sugere que tal posição deva ser reexaminada (DIPELOU \& AKINBOADE, 1982; GODDARD, 1989; CARPENTER et. al., 1990).

VARGAS, et al. (1994 - Informe verbal), visitando tanto a periferia da cidade quanto zonas mais favorecidas de Porto Alegre confirmaram a facilidade com que estes artrópodos se instalam nos terrenos e nas construções humanas, o que já foi constatado também por ARAGÃO (1936). As vilas populares de Porto Alegre são as mais atingidas pela infestação por R. sanguineus, e, apesar de não terem sido documentados casos de parasitismo humano em Porto Alegre até agora, vários depoimentos de profissionais e usuários do sistema de saúde da rede municipal desta cidade tem relatado a sua ocorrência especialmente em crianças.

GODDARD (1989) e CARPENTER $\boldsymbol{e t} \boldsymbol{a l}$. (1990) também observaram que, na maioria dos casos de parasitismo humano, foram as crianças as mais atingidas pelo carrapato, sugerindo que isto deva ocorrer pelo maior contato destas com os cães domésticos, podendo o homem segundo FELDMAN - MUHSAM (1986) ser atacado por todos os instares parasitários do $\boldsymbol{R}$. sanguineus. 
Como o cão está mais exposto que o homem aos carrapatos, ele também pode auxiliar na localização dos focos de Febre Maculosa e da Febre Botonosa (FENG et al., 1979; SMITH et al., 1983), e nas Américas, somente a $\boldsymbol{R}$. rickettsii foi identificada até agora (PLANK et al., 1979).

No Brasil, a Febre Maculosa foi denominada inicialmente de Tifo Exantemático de São Paulo (MONTEIRO, 1931). Hoje está identificada à Febre Maculosa das Montanhas Rochosas, graças aos trabalhos de MONTEIRO (1933) no Brasil e PARKER \& DAVIS (1933) e DYER (1933) nos Estados Unidos. Segundo MONTEIRO \& TIRIBA (1976), os principais vetores da Febre Maculosa Brasileira são: $\boldsymbol{A}$. cajennense, A. aureolatum, A. ovale, A. brasiliensis e A. cooperi. A doença tem caráter endêmico, geralmente em zona rural e está relacionada com o aumento do número de carrapatos transmissores na região.

De acordo com FLECHTMANN (1990), o A. ovale é uma espécie comum dos animais selvagens no Brasil (onças, cervídeos, coatis, raposas, antas, etc.) e que, com o tempo, adaptou- se aos cães domésticos nas zonas rurais, sendo hoje um dos carrapatos comuns nesse animal.

O A. aureolatum também se encontra amplamente distribuido no Brasil, tendo sido observado parasitando o cão, a cabra, o boi, o gambá, o veado, a capivara, o coati e vários canídeos silvestres (FLECHTMANN, 1990); é bastante comum no cão, sendo a espécie mais freqüente nesse hospedeiro no estado do Paraná (RIBEIRO, 1970/1971). Segundo OKOH (1983) os cães de rua estão sob maior risco de tornarem-se infectados e infestados com endo e ectoparasitos respectivamente. DIPEOLU \& AKINBOADE (1982) também chamaram a atenção sobre a importância dos cães de rua na disseminação e na manutenção dos carrapatos entre eles.

Considerando-se o potencial da transmissão de doenças aos animais e o homem pelos carrapatos que parasitam os cães, o grande número de cães vadios que perambulam pelas ruas da cidade e o aumento de casos de infestações por carrapatos em cães, é importante conhecer com precisão as espécies de carrapatos que parasitam os cães de rua da área urbana de Porto Alegre assim como suas prevalências nestes animais. Com isso, poder-se-á estimar a extensão do problema na região e adotar medidas de controle adequadas à(s) espécie(s) de carrapato(s) envolvida(s).

\section{MATERIAIS E MÉTODOS}

No período de maio/93 a abril/94 foram examinados 450 cães, capturados nas ruas da área urbana de Porto Alegre (PORTO ALEGRE, 1994) pelo Serviço de Apreensão de Animais, Centro de Controle de Zoonoses (CCZ), Centro de Vigilância em Saúde (CVS), Secretaria Municipal de Saúde (SMS), Prefeitura Municipal de Porto Alegre (PMPA) e mantidos no Canil Municipal do mesmo órgão, para se proceder a coleta dos carrapatos que os infestavam.

O total de carrapatos obtidos no corpo de cada animal, pela inspeção visual e táctil de todas as suas regiões, foram acondicionados em frascos de vidro individualizados contendo álcool $70^{\circ} \mathrm{GL}$ e identificados no Setor de Entomozooses, Faculdade de Veterinária, Universidade Federal do Rio Grande do Sul um a um, por instar e por espécie, com o auxílio de um estereomicroscópio.

Para a identificação das espécies de carrapatos encontradas nos cães, utilizou-se as chaves de ARAGÃO \& FONSECA (1961) e de JONES et al. (1972) e para os instares de $R$. sanguineus, as descrições de PEGRAM et al. (1987).

\section{RESULTADOS E DISCUSSÃO}

Dos 450 cães de rua de Porto Alegre examinados no período do estudo, $236(52,44 \%)$ estavam parasitados por carrapatos. As espécies de carrapatos encontradas nos animais foram somente duas, ou seja, R. sanguineus e A. aureolatum, concordando com os achados de OLIVEIRA \& RIBEIRO (1982/1983). Uma possível explicação para não ter sido encontrado $A$. ovale parasitando os cães de rua de Porto Alegre, como o encontrou CÔRREA (1947), é o fato dessa espécie ser mais adaptada aos animais das áreas rurais (MASSARD et $\boldsymbol{a l}$., 1981; FLECHTMANN, 1990). Com relação ao $\boldsymbol{A}$. aureolatum, os resultados deste trabalho diferem daqueles relatados por MASSARD et al. (1981), os quais inviabilizam a possibilidade de se encontrar esta espécie na área urbana. No entanto, uma possibilidade, em vista dos resultados obtidos, é que esta espécie possa ocorrer nesta cidade em áreas urbanas pouco populosas ou situadas nos limites destas com a zona rural. As duas espécies de carrapatos ocorreram isoladas ou simultaneamente nos animais.

Considerando-se o tipo de infestação e as espécies de carrapatos envolvidas, as prevalências foram de $93,22 \%$ para os cães parasitados com $\boldsymbol{R}$. sanguineus, 2,97\% para aqueles com A. aureolatum e de $3,81 \%$ para os infestados simultaneamente com $\boldsymbol{R}$. sanguineus e A. aureolatum. Tais índices são bastante diferentes dos obtidos por CORRÊA (1947) e OLIVEIRA \& RIBEIRO (1982/ 1983), evidenciando um aumento do número de cães parasitados em Porto 
Alegre por essas espécies e, em especial, por $\boldsymbol{R}$. sanguineus.

Dos 5294 carrapatos colhidos nos animais do estudo, $99,43 \%$ pertenciam a espécie $\boldsymbol{R}$. sanguineus e somente $0,57 \%$ a A. aureolatum. A média de carrapatos/animal entre os cães de rua parasitados foi de 22,42 exemplares, no entanto, $58,80 \%$ dos cães parasitados apresentavam de um a cinco carrapatos.

MUMCUOGLU et al. (1993b), apesar de terem encontrado diferenças quanto a composição das espécies e aos índices parasitários dos cães infestados com carrapatos em dois kibutz israelenses, também observaram que a maioria dos cães parasitados albergava de um a dez carrapatos/animal, resultados estes que se assemelham aos obtidos no presente trabalho.

Foram coletados no período de estudo, entre os cães de rua de Porto Alegre, 104 exemplares de $\boldsymbol{R}$. sanguineus do estágio larval (1,97\%), 1239 do estágio ninfal $(23,54 \%)$ e $3921(74,49 \%)$ do estágio adulto. Tais resultados diferem dos obtidos por KOCH (1982) em Oklahoma e Arkansas (USA) e por MUMCUOGLU et al. (1993a) em Israel, os quais encontraram nos cães infestados com $\boldsymbol{R}$. sanguineus uma proporção de $86,43 \%$ para $13,57 \%$ e de $15 \%$ para $85 \%$ para os estágios imaturos e adultos respectivamente.

Segundo MOREL \& VASSILIADES (1963), o R. sanguineus é capaz de completar o seu ciclo de vida inteiramente nos cães, quando estes convivem com o homem e outros dos seus hospedeiros estão ausentes. Na sua área de distribuição silvestre, normalmente carnívoros ( onde se inclui o cão) e os ungulados são os principais hospedeiros do estágio adulto de $\boldsymbol{R}$. sanguineus, enquanto que os roedores e os insectívoros são os hospedeiros dos estágios imaturos.

No presente trabalho, não foi feito um levantamento envolvendo outros hospedeiros além do cão. Por isso, não se pode afirmar que o baixo índice dos estágios imaturos de $\boldsymbol{R}$. sanguineus verificado nos cães de rua em Porto Alegre (quando comparado ao do estágio adulto) seja devido a infestação de outros hospedeiros, no entanto não se pode também descartar tal possibilidade. Só foram encontrados exemplares de A. aureolatum na fase adulta, o que confirma os resultados de MASSARD et al. (1981), que também registraram este fato.

Devido a importância epidemiológica destas espécies de carrapatos como vetores de doenças, é importante que os Serviços Oficiais de Saúde de Porto Alegre mantenham um monitoramento dos índices de infestação dos cães e do homem por carrapatos e identifiquem quais são exatamente as doenças transmitidas por eles nesta cidade.

\section{CONCLUSÕES}

Os cães de rua de Porto Alegre, RS são parasitados por Rhipicephalus sanguineus e Amblyomma aureolatum, os quais podem infestá-los de forma isolada ou simultânea. O Rhipicephalus sanguineus é o mais freqüente. Atualmente, os cães têm maior risco de adquirir doenças transmitidas por Rhipicephalus sanguineus e Amblyomma aureolatum do que em anos anteriores, na região de Porto Alegre, RS.

\section{AGRADECIMENTOS}

Aos Funcionários do Canil da Prefeitura Municipal de Porto Alegre- Sérgio Ricardo Ferreira Farias (In memorian) e Alexandre Viegas, e a todos os técnicos e estagiários do Centro de Controle de Zoonoses que colaboraram para a realização deste trabalho.

\section{INFORME VERBAL}

VARGAS, C.R.B., FETZER, L.O., WEBER, M.A. Centro de Zoonoses do Centro de Vigilância em Saúde da Secretaria Municipal de Saúde da Prefeitura Municipal de Porto Alegre. Av. João Pessoa, 325. $2^{\circ}$ andar. 90040-000. Porto Alegre, RS, Brasil.

\section{REFERÊNCIAS BIBLIOGRÁFICAS}

ACHA, P.N., SZYFRES, B. Zoonosis y enfermedades transmisibles comunes al hombre y a los animales. 2.ed. Washington: OPS/ OMS, 1986. 989 p.

ARAGÃO, H.B. Ixodidas brasileiros e de alguns países limitrophes. Mem Inst Oswaldo Cruz, Rio de Janeiro, v. 31, p. $759-844,1936$.

ARAGÃO, H.B., FONSECA, F. Notas de ixodologia. VIII Lista e chave para os representantes da fauna ixodológica brasileira. Mem Inst Oswaldo Cruz, Rio de Janeiro, v. 59, n. 2, p. 115130, 1961.

CARPENTER, T.L., McMEANS, M.C., MCHVGH, C.P. Additional instances of human parasitism by the Brown Dog Tick (Acarina: Ixodidae). J Med Entomol, v. 27, p. 10651066, 1990.

CORNACK, K.M., O'ROURKE, P.K. Parasites of sheep dogs in the Charville District, Queensland. Aust Vet J, v. 68, n. 4, p. 149, 1991.

CORRÊA, O. Contribuição e determinação dos índices helmintológico e entomológico de Canis familiaris Linnaeus na cidade de Porto Alegre. Porto Alegre: Continente, 1947. 86 p.

DIPEOLU, O.O. A Survey of the ectoparasitic infestations of dogs in Nigeria. J Small Anim Pract, v. 16, p. 123-129, 1975.

DIPEOLU, O.O., AKINBOADE, O.A. Scavenging dogs and the spread of tick infestation in Nigheria. Int J Zoon, v. 9, n. 2, p. 90-96, 1982. 
ENCINAS GRANDES, A. Ticks of the province of Salamanca (Central/ NW Spain). Prevalence and parasitization intensity in dogs and domestic ungulates. Ann Parasitol Hum Comp, v. 61 , n. 1 , p. $95-107,1986$.

DYER, R. Relationship between Rocky Mountain spotted fever and exanthematic typhus of São Paulo. Pub Health Rep, v. 48 , p. $512,1933$.

FELDMAN- MUHSAM, B. Ixodid ticks attacks on man in Israel: medical implications. Israel J Med Sci, v. 22, n. 1, p. 19-23, 1986

FENG, W.C., MURRAY, E.J., ROSENBERG, G.E. et al. Natural infection of dogs on cape cod with Rickettsia rickettsii. J Clin Microbiol, v. 10, p. 322-325, 1979.

FLECHTMANN, C.A.W. Ácaros de importância médicoveterinária. 3.ed. São Paulo: Nobel, 1990. 192 p.

FREIRE, J.J. Revisão das espécies da familia Ixodidae. Rev Med Vet, v. 8, n. 1, p. 1-16, 1972.

GODDARD, J. Focus of human parasitism by the brown dog tick, Rhipicephalus sanguineus (Acari: Ixodidae). J Med Entomol, v. 26, n. 6, p. 628-629, 1989.

GREENE, C.E., PHILLIPS, R.N. Rocky Mountain spotted fever. In: GREENE, C.E. Clinical microbiology and infectious diseases of the dog and cat. Philadelphia: Saunders, 1984. Cap. 33. p. 562-575.

HORAK, I.G., GUILLARMOD, A.J., MOOLMAN, L.C. et al. Parasites of domestic and wild carnivores. Onderstpoort J Vet Res, v. 54, p. 573-580, 1987.

JONES, E.K., CLIFFORD, C..M., KEIRANS, J.E. et al. The ticks of Venezuela (Acarina: Ixodoidea) with a key to the species of Amblyomma in the western hemisphere. Brigham Young Univ Sci Bull, Biol. Ser. 17, v. 17, p. 1-40, 1972.

KOCH,H.G. Seasonal incidence and attachment sites of ticks (Acari: Ixodidae) on domestic dogs in southeastern Oklahoma and northwestern Arkansas, USA. J Med Entomol, v. 19, n. 3, p. 293-298, 1982.

KRINSKY, W.L. Dermatoses associated with the bites mites and ticks (Arthropoda: Acari). Int J Dermatol, v. 22, n. 2, p. 7591, 1983.

MASSARD, C.A., MASSARD, C.L., REZENDE, H.E.B. et al Carrapatos de cães em áreas urbanas e rurais de alguns estados brasileiros. In: CONGRESSO BRASILEIRO DE PARASITOLOGIA, 6., 1981, Belo Horizonte, MG. Resumos.. Belo Horizonte: Sociedade Brasileira de Parasitologia, 1981. p. 201.

MONTEIRO, J.L. Estudos sobre o tipho exanthemático de S. Paulo. Mem Inst Butantã, São Paulo, v. 6, p. 3, 1931

MONTEIRO, J.L. O "tifo exanthemático de São Paulo" e suas relações com a febre maculosa das Montanhas Rochosas à luz das provas de imunidade cruzada. Bol Soc Med e Cir, São Paulo, v. 17, p. 55, 1933.

MONTEIRO, E.V.L., TIRIBA, A.C. Riquetsioses. In: VERONESI,
R. Doenças infecciosas e parasitárias. 6.ed. Rio de Janeiro: Guanabara Koogan, 1976. Cap. 31, p. 236-249.

MOREL, P.C., VASSIALIADES, G. Les Rhipicephalus du groupe sanguineus: espéces africaines (Acariens: Ixodoidea). Rev Elev Méd Vet Pays Trop, v. 15, n. 4, p. 343-386, 1962.

MUMCUOGLU, K.Y., BURGAN, I., IOFFE-USPENSKY, I. et al. Rhipicephalus sanguineus: Observations on the parasitic stage on dogs in the Negev Desert of Israel. Experim Appl Acarol, v. 17, p. 793-798, 1993a.

MUMCUOGLU, K.Y., FRISH, K., SAROV, B. et al. Ecological studies on the brown dog tick Rhipicephalus sanguineus (Acari: Ixodidae) in Southern Israel and its relationship to spotted fever group Rickettsiae. J Med Entomol, v. 30, n. 1, p. 114-121, 1993b.

$\mathrm{OKOH}$, A.E.J. Canine diseases of public health significance in Nigeria. Int J Zoon, v. 10, n. 1, p. 33-39, 1983.

OLIVEIRA, C.M.B., RIBEIRO, P.B. Espécies de pulgas que parasitam cães em Porto Alegre e suas prevalências mensais Arq Fac Vet UFRGS, Porto Alegre, v. 10-11, p. 29-33, 1982/ 198383.

PARKER, R.R., DAVIS, G.E. Further studies on the relationship of the viruses of Rocky Mountain spotted fever and São Paulo exanthematic typhus. Pub Health Rep, v. 48, p. 859, 1933.

PEGRAM, R.G., KEIRANS, J.E., CLIFFORD, C.M. et al. Clarification of Rhipicephalus sanguineus group (Acari, Ixodoidea, Ixodidae) II. R. sanguineus (Latreille, 1806) and related species. System Parasitol, v. 10, n. 1, p. 27-44, 1987.

PLANK, S.J., TEIXEIRA, R.S., MILANESI, M.L. Febre maculosa em Salvador: descrição de um caso. Rev Med Bahia, Salvador, v. 25 , p. $330-334,1979$.

PORTO ALEGRE. Prefeitura Municipal. Secretaria do Planejamento Municipal. Plano Diretor de Desenvolvimento Urbano de Porto Alegre- $1^{\circ}$ PDDU- Lei Complementar $n^{\circ}$ 43/79. 4.ed. Porto Alegre: CORAG, 1994. 428 p.

RIBEIRO, S.S. Ixodídeos encontrados no cão doméstico no Estado do Paraná. An Fac Med UFPar, Curitiba, v. 13/14, n. 1/2, p. 61-67, 1970/71.

RIBEIRO, V.L.S., WEBER, M.A., FETZER, L.O. et al. Ixodídeos parasitos de cães de rua em Porto Alegre, RS. (Nota prévia). Arq Fac Vet UFRGS, Porto Alegre, v. 22, p. 55-56, 1994.

RIVOSECCHI, L., KHOURI, C., LEZZERINI, C. et al Observazioni su Rhipicephalus sanguineus (Ixodidae) nella periferia di Roma. Riv Parass, v. 41, n. 2, p. 273-276, 1980.

SMITH, R.C., GORDON, J.C., GORDON, S.W. et al. Rocky Mountain spotted fever in an urban canine population. J Am Vet Med Ass, v. 183, p. 1451-1453, 1983.

SWANGO, L.J., BANKEMPER, K.W., KONG, L.I. Infecções bacterianas, riquetsiais, protozoais e outras. In: ETTINGER, S.J. Tratado de medicina interna veterinária (Moléstias do cão e do gato). 3.ed. São Paulo: Manole, 1992. Cap. 46. Seção III: Doenças Infecciosas: p. 277-311. 